\title{
Effectiveness of a community-based osteoporosis education and self-management course: a wait list controlled trial
}

\author{
K. L. Francis • B. L. Matthews • W. Van Mechelen • \\ K. L. Bennell • R. H. Osborne
}

Received: 18 January 2008 / Accepted: 24 November 2008 /Published online: 5 February 2009

(C) International Osteoporosis Foundation and National Osteoporosis Foundation 2009

\begin{abstract}
Summary Osteoporosis is an increasing burden on individuals and health resources. The Osteoporosis Prevention and Self-Management Course (OPSMC) was designed to assist individuals to prevent and manage osteoporosis; however, it had not been evaluated in an Australian setting. This randomised controlled trial showed that the course increased osteoporosis knowledge.

Introduction and hypothesis Osteoporosis is a major and growing public health concern. An OPSMC was designed to provide individuals with information and skills to prevent or manage osteoporosis, but its effectiveness has not previously been evaluated. This study aimed to determine whether OPSMC attendance improved osteoporosis knowledge, self-efficacy, self-management skills or behaviour.

Materials and methods Using a wait list randomised controlled trial design, 198 people ( $92 \%$ female) recruited from the community and aged over 40 (mean age $=63)$ were randomised into control $(n=95)$ and intervention $(n=103)$ groups. The OPSMC consists of four
\end{abstract}

K. L. Francis • B. L. Matthews $\cdot$ K. L. Bennell Centre for Health, Exercise and Sports Medicine, The University of Melbourne,

Parkville, Australia

W. Van Mechelen

Department of Public \& Occupational Health and EMGO Institute, VU University Medical Center,

Amsterdam, The Netherlands

R. H. Osborne ( $\triangle)$

Centre for Rheumatic Diseases, Department of Medicine,

Royal Melbourne Hospital, The University of Melbourne, Parkville, Australia

e-mail: richardo@unimelb.edu.au weekly sessions which run for $2 \mathrm{~h}$ and are led by two facilitators. The primary outcome were osteoporosis knowledge, health-directed behaviour, self-monitoring and insight and self-efficacy.

Results The groups were comparable at baseline. At 6-week follow-up, the intervention group showed a significant increase in osteoporosis knowledge compared with the control group; mean change $3.5(p<0.001)$ on a measure of $0-20$. The intervention group also demonstrated a larger increase in health-directed behaviour, mean change $0.16(p<$ $0.05)$, on a measure of $0-6$.

Conclusion The results indicate that the OPSMC is an effective intervention for improving understanding of osteoporosis and some aspects of behaviour in the short term.

Keyword Evaluation · Knowledge · Osteoporosis ·

Patient education $\cdot$ Self-management

\section{Introduction}

Osteoporosis is a condition where there is a reduction in bone density, which in turn leads to an increased risk of bone fracture. Osteoporosis poses a significant disease and economic burden globally [1, 2], with an estimated nine million osteoporotic fractures worldwide in 2000 [1]. Pain as a consequence of fracture can be long term, and frequently, there is an associated loss of mobility [3]. Feelings of helplessness, anxiety and fear of future fractures are all potential negative psychological outcomes resulting from osteoporotic fractures or even low bone density [4]. Although associated with ageing, osteoporosis and its associated fractures are not an inevitable part of the ageing process. 
For chronic conditions like osteoporosis, the health care system needs to focus on disease prevention through education and self-management support. Formalised selfmanagement programs are designed to complement usual care [5] and as such are particularly appropriate health education initiatives for people with chronic disease. Individuals fare better when they can competently manage day to day fluctuations in their health [6] as opposed to relying solely on health professionals to manage their chronic disease(s). Effective health education interventions have been identified as those which provide knowledge and skills and foster confidence as opposed to dependency [7].

Given the number of people suffering from osteoporosis, health promotion programs are increasingly seen as a potentially cost-effective way of preventing the development or progression of this disease. The knowledge and skills provided through such programs may lead to behavioural change such as increasing calcium consumption and weight-bearing exercise [8], which are key modifiable factors known to prevent osteoporosis [9-13].

The Osteoporosis Prevention and Self-Management Course (OPSMC) was developed in Australia to increase understanding of the disease and hence decrease anxiety and to promote healthy behaviours likely to prevent the disease or facilitate effective management. In order for behaviour change to occur, individuals need to be informed about the area of health which the behaviour change is targeted. Research has shown that there is a lack of knowledge of osteoporosis within the Australian community $[14,15]$. Although knowledge alone is usually insufficient to elicit long-term behaviour change, it is a vital component [16-18].

Data regarding the effectiveness of self-management programs are varied. Importantly, meta-analyses demonstrated reductions in clinical measures such as symptoms in people with hypertension, diabetes and arthritis [19, 20]. Other more proximal or intermediate outcomes such as selfmanagement behaviours or effective interactions with the healthcare system were less commonly measured and not subjected to meta-analyses. Recent Australian research into the effectiveness of self-management education interventions for people with chronic diseases suggest that approximately one third of participants received substantial improvements in self-management behaviours from a wide variety of interventions [21].

Previous studies that have focused on osteoporosis in people aged over 40 years (the target audience of the OPSMC) has indicated that interventions ranging from several weeks to single sessions can result in increased knowledge about osteoporosis $[17,18]$. Osteoporosis selfmanagement interventions have also been associated with increased calcium consumption and engagement in more weight-bearing exercise $[8,17,22]$. An osteoporosis self- management course in the USA-Choices For Better Bone Health - promotes positive health behaviours by providing disease-specific information and behavioural skills for those at risk or with osteoporosis [23].

Many governments are investing in community-wide self-management education interventions for a wide range of chronic diseases [24, 25]. However, limited robust and high-quality information about the effectiveness of these programs is available. The OPSMC is an established Australian self-management course for both men and women aged 40 years or older who have osteoporosis, osteopenia or may be at risk of developing osteoporosis. However, this course had not been formally evaluated. As the OPSMC presents specific osteoporosis information as well as integrating techniques to enhance self-management, it has the potential to make a major ongoing contribution to the management of this disease.

This study was a randomised controlled trial to determine whether the OPSMC leads to improvements in osteoporosis knowledge, self-management skills and self-efficacy for calcium consumption and exercise engagement. It was hypothesised that OPSMC attendance would generate increased knowledge about osteoporosis, substantial improvements in health-directed behaviour and self-monitoring and greater self-efficacy with regard to engaging in exercise and calcium consumption.

\section{Materials and methods}

\section{Participants}

Participants were recruited from the community via print media, the Internet and flyers. The inclusion criteria were: age 40 years or over, able to read and write in English, as well as self-reported diagnosis of osteoporosis or osteopenia, or having any of the following risk factors for osteoporosis: family history of osteoporosis, having sustained a fracture after a minor bump or fall, female gender and history of smoking. Participants were excluded from the study if they had completed an osteoporosis education program or similar self-management course previously.

The project was approved by the Human Research Ethics Committee at The University of Melbourne. After telephone screening, participants who met the eligibility criteria and provided written informed consent were randomly allocated to either the 'control' or 'intervention' group using a computer-generated random number list before the initial surveys were sent out. Separate lists were generated for each of the 17 course locations. Those in the control group completed surveys at baseline and 6 weeks later. They were given the option of attending the OPMSC after completing the second survey. The intervention group 
completed their surveys at baseline and then attended an OPSMC. This group completed their follow-up surveys approximately 2 weeks after the last course session (6 weeks after their baseline survey).

\section{Intervention}

The OPSMC was conducted once a week as a 2- to 2.5$\mathrm{h}$ session over four consecutive weeks. Two trained leaders, who were either health professionals or lay people with a personal or professional interest in the self-management of osteoporosis, delivered the sessions.

The course covers a variety of topics (Table 1), often introduced more than once, so that if a session is missed, a participant will still encounter at least some knowledge about those uncompleted sections. Participants are provided with a course manual 'Everybody's Bones' [26].

In the first session, the participants identify what osteoporosis means to each of them and what factors motivated them to attend the course. This is followed by an introduction to self-management and osteoporosis (basic bone physiology and the consequences of osteoporosis), making sure myths (such as osteoarthritis and osteoporosis are similar conditions) are dispelled. The session also covers the benefits of weight-bearing physical activity and the role of different forms of exercise, followed by how to start a personal exercise regime. The last activity is action planning whereby participants (and leaders) set goals for the week to help prevent or manage osteoporosis.

The second and subsequent sessions start with feedback and problem solving arising from the action plans. Session two continues with an examination of the risk factors for osteoporosis, separating them into those which participants have control over and those they do not. The next activity reinforces the problem solving process and the importance of exercise. Participants identify common obstacles to exercise and potential solutions. Calcium is also reviewed

Table 1 Topics covered in the OPSMC

Overview of self-management
Detailed description of osteoporosis and bone remodeling process
Risk factors for osteoporosis
Medications (review of current osteoporosis medications)
Action planning
Pain management/cognitive symptom management
Feedback and problem solving
Emotions-anger, fear, frustration
Exercise
Nutrition (focus on calcium and vitamin D)
Falls prevention
Posture
Informing your healthcare team/working with health professionals
Looking to a stronger future

Overview of self-management

Detailed description of osteoporosis and bone remodeling process

Risk factors for osteoporosis

Medications (review of current osteoporosis medications)

Action planning

Feedback and problem solving

Emotions - anger, fear, frustration

Exercise

Nutrition (focus on calcium and vitamin D)

ention

Informing your healthcare team/working with health professionals Looking to a stronger future from the recommended levels and how to augment diet to meet the required level through to suggestions for lactoseintolerant individuals. Calcium supplements and the role of dietary factors in the process of calcium absorption are also discussed. The session closes with action planning.

In session 3, the next knowledge focus is bone density measurement. It includes topics such as different measurement techniques and who should be tested. The next area, managing strong emotions, is presented as relevant to general health and well-being and not to be compartmentalised only for osteoporosis. Medications for osteoporosis, how they work and whom they might be most suitable for are also covered. Emphasis is placed on the importance of effective communication with healthcare professionals. Positive working relationship with the healthcare team are encouraged, suggesting use of the acronym PART — Prepare a list of questions, Ask questions, Repeat the key points, Take action on the advice given or explain why you cannot do what is asked of you. Effective communication techniques are also highlighted. Again, the session ends with action planning.

The fourth session includes a demonstration of good posture and safe bending /lifting practices. This leads to a discussion on falls prevention in the home. A review of pain management options follows, covering cognitive techniques, medications and physical techniques. The role of outside support and local support groups is then discussed. In the fourth session, action planning looks at longer term goals. The last activity reviews the topics covered over the previous 4 weeks.

\section{Outcome measures}

The primary outcome measure was knowledge change, assessed by the Osteoporosis Knowledge Assessment Test (OKAT) [24]. The OKAT consists of 20 questions requiring a true, false or don't know response about the definition of osteoporosis, risk factors, consequences, treatments and the role of diet and exercise. Participants receive one mark for each correct answer and nothing for a 'don't know' or an incorrect answer, with OKAT scores ranging from 0 to 20 . This questionnaire was developed in Australia and has been validated in a study with Australian women aged 45 years or younger [27]. For items on the OKAT in the current study, the Cronbach alpha was 0.69 .

The second measure employed in this study was the Health Education Impact Questionnaire (HeiQ), which measures eight domains to determine the proximal outcomes of self-management courses. Each domain has a score ranging from 1 to 6 which corresponds to a Likert scale of strongly disagree to strongly agree. Each item and each domain of the HeiQ have been rigorously tested. A confirmatory factor analysis was conducted to reveal that 
each domain was a homogenous construct with Cronbach alpha and factor structures indicating good to excellent psychometric properties. It is currently used in many countries as a quality and monitoring tool and as a primary measure of proximal outcomes. Further details are available (http://www.heiq.org.au). Reliability for each domain ranged from 0.7 to $0.89[21,28]$. The domains are:

1. Positive and active engagement in life-This covers motivation to be active and embodies the notion of participants in self-management/health education programs engaging or reengaging in life-fulfilling activities. An example item includes: I am doing interesting things in my life. For items in this domain in the current study, the Cronbach alpha was 0.81 .

2. Health-directed behaviour-This construct is similar to the first in that it relates to lifestyle and covers tangible healthful behaviours. An example item includes: $O n$ most days of the week, I do at least one activity to improve my health (e.g., walking, relaxation, exercise). For items in this domain in the current study, the Cronbach alpha was 0.88 .

3. Skill and technique acquisition-This aims to capture the knowledge-based skills and techniques (including the use of aids) needed to help manage and cope with disease-related symptoms and health problems. An example item includes: When I have symptoms, I have the skills that help me cope. For items in this domain in the current study, the Cronbach alpha was 0.72 .

4. Constructive attitudes and approaches-This construct is embodied by the statement "I am not going to let this disease control my life" and includes an individual's views the impact of their condition(s) on their life. Example items includes: I try not to let my health problems stop me from enjoying life and I feel I have a very good life even when I have health problems. For items in this domain in the current study, the Cronbach alpha was 0.76 .

5. Self-monitoring and insight-This captures the individuals' ability to monitor their condition and their physical and/or emotional responses that lead to insight and appropriate actions to self-manage. Examples item includes: When I have health problems, I have a clear understanding of what I need to do to control them and I know what things can trigger my health problems and make them worse. For items in this domain in the current study, the Cronbach alpha was 0.65 .

6. Health services navigation-This is concerned with an individual's understanding of and ability to confidently interact with a range of health organisations and health professionals. An example item includes: I communicate very confidently with my doctor about my healthcare needs. For items in this domain in the current study, the Cronbach alpha was 0.77 .
7. Social integration and support-This aims to capture the positive impact of social engagement and support that evolves through interaction with others. An example item includes: If I need help, I have plenty of people I can rely on. For items in this domain in the current study, the Cronbach alpha was 0.83 .

8. Emotional wellbeing-This measures overall negative affective responses to illness, including anxiety, anger and depression (which are attributed to the illness). Example items include: If I think about my health, I get depressed and I get upset when I think about my health. For items in this domain in the current study, the Cronbach alpha was 0.84 .

The Osteoporosis Self-Efficacy Scale (OSES) was used to assess participant confidence and their ability to engage in appropriate physical activity (type and duration) to prevent further bone loss and to obtain the recommended amount of dietary calcium. Three OSES scores were recorded, one for calcium, one for exercise and a total combined score for these two subscales. There are ten questions in the exercise subscale and 11 in the calcium subscale. For each statement, the participant places a mark on a $10-\mathrm{cm}$ visual analogue scale to indicate their level of endorsement of each item. This results in a score out of ten for each item that is then reduced to a scale of $0-10$ for the entire scale. The OSES was validated in a study of 201 women aged 35 to 95 years, mean age of 56 years [29]. For all items on the OSES in the current study, the Cronbach alpha was 0.95 (whilst for the exercise and calcium items specifically, the Cronbach alphas were 0.92 and 0.96, respectively).

\section{Data analysis}

Missing values in each variable were initially examined to determine if they were randomly distributed. The 'last value carried forward' approach was used for the missing data, that is, the participant's previous score was used when the follow-up data was missing ( $n=27 ; 14 \%$ of the sample: 14 participants from the intervention group and 13 from the control group). This conservative approach is in keeping with the null hypothesis that a person's score will not change over time.

Baseline group differences between groups were tested via $t$ tests or equivalent non-parametric tests. Analysis of covariance, adjusting for baseline scores and baseline group differences, was used to examine whether an intervention effect was present. Cohen's effect size was determined by subtracting the mean change scores for the intervention and control group and dividing by the pooled standard deviation. 


\section{Results}

The sample consisted of 198 participants (103 in the intervention group and 95 in the control group), the mean age was 63 years, and $92 \%$ were female. No baseline differences were found between the groups for age, gender, highest level of education attained, country of birth, language spoken at home, osteoporosis status, smoking status, private health insurance status, employment status, number of chronic conditions and the key outcome measures (Table 2).

\section{Knowledge}

At follow-up, the intervention group had a higher mean knowledge score compared to the control group: 13.49 versus 10.05, respectively $(p<0.001)$. The intervention group showed an improvement in knowledge scores of 3.58 (36\% increase), whilst the control group score changed by only $0.05(0.5 \%$; Table 3$)$. This effect size was large, with a Cohens $D$ value of 1.32 .

\section{HeiQ}

At follow-up, when compared with the control group, the intervention group had larger improvements in healthdirected behaviour [mean (SD) difference between groups; $0.16(0.74), p=0.020]$, positive and active engagement in life [0.14 $(0.51), p=0.048]$, skill and technique acquisition $[0.22(0.56), p=0.006]$ and social integration and support [0.17 (0.55), $p=0.033]$.

\section{Self-efficacy}

At follow-up, there were no significant differences between the control and intervention groups for the calcium, exercise and total OSES scores (Table 3).

\section{Discussion}

The results of this study indicate that the OPSMC generates valuable impacts on participants through increasing knowledge of osteoporosis by approximately $36 \%$ compared with the control group. This observation was supported by a larger increase in skills and techniques in the intervention group compared with the controls, and the OPSMC led to small increases in health-directed behaviour, positive and active engagement in life and social integration and support.

The finding of an increase in participant knowledge is consistent with other community osteoporosis interventions $[28,30,31]$. The baseline OKAT score for the current study (mean score 10) was greater than that found in a study of younger women aged 25-45 years (mean score for the four groups 8.8) [28]. These women reported a post-OPSMC knowledge gain at 6 weeks of approximately 4.0 units $(45 \%)$ and of 2.8 units (31\%) at 2-year follow-up [22]. The current study's average 3.6-unit increase in knowledge following the OPSMC is of a similar magnitude to that seen in the other study (36\%, range $28-43 \%$ ) [22].

An increase in knowledge of osteoporosis following the OPSMC is a valuable finding. Prior to attending the course,

Table 2 Comparison of OKAT, OSES and HeiQ measures at baseline for the control and intervention groups

\begin{tabular}{lll}
\hline & Control $(N=95)$ & Intervention $(N=103)$ \\
\cline { 2 - 3 } & Mean $(\mathrm{SD})$ & Mean (SD) \\
\hline OKAT & & \\
Knowledge & $10.00(3.65)$ & $9.91(3.13)$ \\
OSES & & \\
Exercise self-efficacy & $7.58(1.83)$ & $7.25(2.09)$ \\
Calcium self-efficacy & $7.89(1.67)$ & $7.85(2.02)$ \\
Total self-efficacy & $7.74(1.45)$ & $7.56(1.83)$ \\
HeiQ & & \\
Health-directed behaviour & $4.62(1.22)$ & $4.71(1.06)$ \\
Self-monitoring and insight & $4.75(0.61)$ & $4.80(0.52)$ \\
Positive and active engagement in life & $4.93(0.76)$ & $4.85(0.71)$ \\
Skill and technique acquisition & $4.35(0.71)$ & $4.39(0.76)$ \\
Constructive attitudes and approaches & $5.12(0.57)$ & $5.03(0.59)$ \\
Health service navigation & $4.69(0.83)$ & $4.69(0.73)$ \\
Social integration and support & $4.26(0.84)$ & 0.83 \\
Emotional well-being & $4.46(1.08)$ & $4.32(0.94)$ \\
\hline
\end{tabular}

Scores for OKAT range from 0 to 20, each item of the OSES range from 0 to 10 and each domain of the HeiQ range from 1 to 6 
Table 3 Comparison of the change in OKAT, OSES and HeiQ scores (baseline to follow-up) between the control and intervention groups for the primary outcome measures

\begin{tabular}{|c|c|c|c|c|c|c|}
\hline \multirow[b]{2}{*}{ Change } & \multirow{2}{*}{$\begin{array}{l}\text { Control } \\
(N=95) \\
\text { Mean (SD) }\end{array}$} & \multicolumn{2}{|c|}{ Intervention $(N=103)$} & \multirow[b]{2}{*}{$\begin{array}{l}95 \% \\
\text { Confidence } \\
\text { Interval }\end{array}$} & \multicolumn{2}{|c|}{$\begin{array}{l}\text { Analysis of } \\
\text { covariance }^{\text {a }}\end{array}$} \\
\hline & & Mean (SD) & $\begin{array}{l}\text { Mean difference between change } \\
\text { scores (SD) }\end{array}$ & & $P$ value & Cohen's $D^{\mathrm{b}}$ \\
\hline \multicolumn{7}{|l|}{ OKAT } \\
\hline Knowledge & $0.05(2.14)$ & $3.58(3.10)$ & $3.53(2.68)$ & $2.768-4.272$ & 0.000 & 1.32 \\
\hline \multicolumn{7}{|l|}{ OSES } \\
\hline Exercise self-efficacy & $0.00(1.43)$ & $0.29(1.79)$ & $0.29(1.63)$ & $-0.171-0.743$ & 0.711 & 0.18 \\
\hline Calcium self-efficacy & $0.10(1.87)$ & $0.25(2.31)$ & $0.15(2.11)$ & $-0.447-0.738$ & 0.582 & 0.07 \\
\hline Total self-efficacy & $0.05(1.32)$ & $0.28(1.82)$ & $0.22(1.60)$ & $-0.231-0.669$ & 0.583 & 0.14 \\
\hline \multicolumn{7}{|l|}{ HeiQ } \\
\hline Health-directed behaviour & $0.04(0.60)$ & $0.20(0.85)$ & $0.16(0.74)$ & $0.007-0.424$ & 0.020 & 0.21 \\
\hline Self-monitoring and insight & $0.01(0.45)$ & $0.13(0.46)$ & $0.11(0.46)$ & $-0.015-0.241$ & 0.057 & 0.25 \\
\hline $\begin{array}{l}\text { Positive and active } \\
\text { engagement in life }\end{array}$ & $-0.03(0.52)$ & $0.11(0.51)$ & $0.14(0.51)$ & $0.001-0.288$ & 0.048 & 0.28 \\
\hline Skill and technique acquisition & $0.03(0.54)$ & $0.25(0.57)$ & $0.22(0.56)$ & $0.063-0.375$ & 0.006 & 0.39 \\
\hline $\begin{array}{l}\text { Constructive attitudes and } \\
\text { approaches }\end{array}$ & $-0.07(0.43)$ & $0.05(0.47)$ & $0.12(0.45)$ & $-0.002-0.252$ & 0.054 & 0.28 \\
\hline Health service navigation & $-0.02(0.54)$ & $0.10(0.54)$ & $0.11(0.54)$ & $-0.037-0.266$ & 0.138 & 0.21 \\
\hline Social integration and support & $-0.06(0.53)$ & $0.11(0.56)$ & $0.17(0.55)$ & $0.014-0.320$ & 0.033 & 0.31 \\
\hline Emotional well-being & $0.01(0.62)$ & $0.01(0.78)$ & $0.00(0.71)$ & $-0.196-0.201$ & 0.978 & 0.00 \\
\hline
\end{tabular}

${ }^{a}$ Covariants included education level, age, country of birth, gender, baseline score

${ }^{\mathrm{b}}$ Cohen's $D$ refers to the effect size with magnitudes classified as follows small $0.20-0.49$, medium $0.50-0.79$ and large $>0.8$

almost half of the participants did not know that osteoporosis could be asymptomatic. Importantly, in the intervention group, only $6 \%$ answered this incorrectly at follow-up. Other research into osteoporosis knowledge indicated that only $33 \%$ to $36 \%$ of the sample was able to correctly answer (true or false) to the statement that osteoporosis always causes pain [32].

Self-efficacy was expected to increase following course completion, with the anticipation of participants having greater confidence in their ability to carry out disease prevention and management behaviours as a result of increased understanding and the development of new skills to tackle the health- and well-being-issues-associated chronic conditions. However, the current data on self-efficacy measured by the OSES showed no difference as a result of the intervention. The findings from other studies with regard to self-efficacy change have been inconsistent $[22,28,31$, 32]. The lack of change may again be as a result of increased understanding of osteoporosis and response shift, as participants may have realised that the type of exercise they were doing in the past was not contributing to bone strengthening. The knowledge and HeiQ change results imply that although the self-efficacy scale did not suggest change, the participants still obtained self-management benefits.

A potential limitation of the research is that those who chose to be involved in the trial may already have had an interest in health and a desire to improve, and the findings may therefore not be indicative of the changes likely to occur with the general population of specific or multiple chronic condition sufferers. Despite this concern, recruitment for similar courses is also often through self-selection rather than, for example, marketing targeted to those most likely to benefit. Viewed in this light, the sample and style of recruitment is a realistic reflection of how course attendees are recruited in most settings. An advantage that arises from using people based on their own interest in a course is that they are usually more likely to attend all sessions and therefore receive the greatest benefit from the allocated health resources. The results of this study suggest that the OPSMC is effective even in a reasonably motivated sample of participants where a ceiling effect may be operating. It is possible that the program is even more effective in unselected samples (e.g. from the hospital outpatient setting) where participants may have greater capacity to benefit, although it may also be less effective in a less motivated group of attendees.

A further limitation of this study is that the wait list control group were not blinded to their group assignment and it is possible that this may have biased the results in favour of the intervention group. Furthermore, the short follow-up period of 2 weeks after the completion of the 4week intervention is also a potential limitation. However, 
previous studies have demonstrated that knowledge and skills gained from osteoporosis specific courses are not transient and can be sustained for at least 2 years $[8,22]$. Other self-management programs have also shown that the benefits of course participation can be sustained for multiple years $[33,34]$. Given that reasonable evidence on the course's effectiveness has been established in the current study, further research should focus on longer term follow-up, looking at behavioural change and reduction in falls and fractures. It is recommended that future long-term studies also assess change in calcium and vitamin D consumption, amount of weight-bearing exercise and number of falls as a result of OPSMC participation.

\section{Conclusion}

This randomised controlled trial is a major step in establishing the role for the OPSMC for managing and preventing osteoporosis in people over 40 years of age within the community. With the growing burden of diseases such as osteoporosis, the OPSMC has a unique role in disease prevention and management, as it is highly diseasespecific. It caters for people who have the condition as well as those who are at risk.

The OPSMC has a positive effect on participants' knowledge of osteoporosis and health-directed behaviour The course appears to cater to the needs of people at risk of, as well as people with, osteoporosis. Osteoporosis is an increasingly prevalent and costly chronic disease, and programs such as the OPSMC which encourage selfmanagement should be widely promoted, particularly to those people who are most likely to benefit.

Conflicts of interest No disclosures or conflict of interests to report.

\section{References}

1. Johnell O, Kanis JA (2006) An estimate of the worldwide prevalence and disability associated with osteoporotic fractures. Osteoporos Int 17:1726-1733

2. Sambrook P, Cooper C (2006) Osteoporosis. Lancet 367:20102018

3. Gass M, Dawson-Hughes B (2006) Preventing osteoporosisrelated fractures: an overview. Am J Med 119:3S-11S

4. Gold D (2005) Quality of life and its measurement in osteoporosis. In: Maricic M, Gluck OS (eds) Bone disease in rheumatology. Lippincott Williams \& Wilkins, Philadelphia, PA, pp 67-79

5. Lorig K, Gonzalez V, Laurent D; Stanford Patient Education Research Centre (1999) The chronic disease self-management program leaders manual. Palo Alto, CA

6. Conn V, Russell C (2005) Self-management of chronic illnesses among aging adults. J Gerontol Nurs 31:4
7. Wagner EH (2000) The role of patient care teams in chronic disease management. Br Med J 320:569-572

8. Pearson JA, Burkhart E, Pifalo WB, Palaggo-Toy T, Krohn K (2005) A lifestyle modification intervention for the treatment of osteoporosis. Am J Health Promot 20:28-33

9. Burckhardt P (2004) Nutrition and osteoporosis. In: Sambrook PN, Geusens P, Lindsay R (eds) Osteoporosis in clinical practice. 2nd edn. Springer, USA, pp 187-192

10. Wark J, Nowson C (2003) Calcium supplementation: the bare bones. Aust Prescr 26:126-127

11. Delaney MF (2006) Strategies for the prevention and treatment of osteoporosis during early postmenopause. Am J Obstet Gynecol 194:S12-S23

12. Kannus P, Uusi-Rasi K, Palvanen M, Parkkari J (2005) Nonpharmacological means to prevent fractures among older adults. Ann Med 37:303-310

13. Sambrook PN, Geusens P (2004) Normal skeletal structure and function. In: Sambrook PN, Geusens P, Lindsay R (eds) Osteoporosis in clinical practice. 2nd edn. Springer, USA, pp 3-7

14. Eisman J, Clapham S, Kehoe L (2004) Osteoporosis prevalence and levels of treatment in primary care: the Australian bone care study. J Bone Miner Res 19:1969-1975

15. Laslett LL, McNeil JD, Lynch J (2004) Patient education-the forgotten link in managing osteoporosis. Aust Fam Physician $33: 121-124$

16. Coates VE, Boore JRP (1995) Self-management of chronic illness: implications for nursing. Int J Nurs Stud 32:628-640

17. Conner M, Norman P (2005) Predicting health behaviour: a social cognition approach. In: Conner M, Norman P (eds) Predicting health behaviour: research and practice with social cognition models. 2nd edn. Open University Press, New York, pp 1-28

18. Tanner EK (2004) Chronic illness demands for self-management in older adults. Geriatr Nurs 25:313-317

19. Chodosh J, Morton SC, Mojica W, Maglione M, Suttorp MJ, Hilton L, Rhodes S, Shekelle P (2005) Improving patient care. Meta-analysis: chronic disease self-management programs for older adults. Ann Intern Med 143:427-438

20. Warsi A, LaValley MP, Wang PS, Avorn J, Solomon DH (2003) Arthritis self-management education programs a meta-analysis of the effect on pain and disability. Arthritis Rheum 48:2207-2213

21. Nolte S, Elsworth GR, Sinclair AJ, Osborne RH (2006) The extent and breadth of benefits from participating in chronic disease self-management courses: a national patient-reported outcomes survey. Patient Educ Couns 65:351-360

22. Winzenberg TM, Oldenburg B, Frendin S, De Wit L, Jones G (2005) Effects of bone density feedback and group education on osteoporosis knowledge and osteoporosis self-efficacy in premenopausal women. J Clin Densitom 8:95-103

23. Gold DT, Silverman SL (2004) Osteoporosis self-management: choices for better bone health. South Med J 97(6):551-554

24. Australian Government Department of Health and Ageing (2006) COAG health services-promoting good health, prevention and early intervention. Fact sheet. Commonwealth Government, Canberra. http://www.health.gov.au/internet/budget/publishing. nsf/Content/budget2006-hfact37.htm (accessed May 2006)

25. Australian Government (2006) Budget. Part 2: expense measures. Canberra: Commonwealth Government. http://www.budget.gov. au/2006-07/bp2/download/bp2_expense.pdf (accessed May 2006)

26. Osteoporosis Victoria (2001a) Everybody's bones, 3rd edn. Arthritis Foundation of Victoria, Melbourne, Australia

27. Winzenberg TM, Oldenburg B, Frendin S, Jones G (2003) The design of a valid and reliable questionnaire to measure osteoporosis knowledge in women: the Osteoporosis Knowledge Assessment Tool (OKAT). BMC Musculoskelet Disord 4:17

28. Osborne R, Whitfield K, Elsworth G (2007) The health education impact questionnaire (HeiQ): An outcomes and evaluation measure 
for patient education and self-management interventions for people with chronic conditions. Patient Educ Couns 66(2):192-201

29. Horan M, Kim K, Gendler P (1993) Development and evaluation of osteoporosis self-efficacy scale. Paper presented at the Paper presented at the Midwest Nursing Researching Society Conference, Cleveland, $\mathrm{OH}$

30. Kulp JL, Rane S, Bachmann G (2004) Impact of preventive osteoporosis education on patient behavior: immediate and 3month follow-up. Menopause 11:116-119

31. Brecher LS, Pomerantz SC, Synder BA, Janora DM, KlotzbachShimomura KM, Cavaleri TA (2002) Osteoporosis prevention project: a model multidisciplinary educational intervention. J Am Osteopath Assoc 102:327-335
32. Solomon DH, Finkelstein JS, Polinski JM, Arnold M, Licari A, Cabral D, Canning C, Avorn J, Katz JN (2006) A randomized controlled trial of mailed osteoporosis education to older adults. Osteoporos Int 17:760-767

33. Lorig KR, Ritter P, Stewart AL, Sobel DS, Brown BW Jr, Bandura A, Gonzalez VM, Laurent DD, Holman HR (2001) Chronic disease self-management program: 2-year health status and health care utilization outcomes. Med Care 39: $1217-1223$

34. Osborne R, Wilson T, Lorig K, McColl G (2007) Does selfmanagement lead to sustainable health benefits in people with arthritis? A two-year transition study of 452 Australians. J Rheumatol 34:1112-1117 